\title{
HYPOCONTINUITY OF MULTIPLICATION ON THE CLIFFORD ALGEBRA OF AN INFINITE-DIMENSIONAL TOPOLOGICAL VECTOR SPACE
}

\author{
ROBERT A. HABERSTROH
}

ABSTRACT. Given a quadratic form on an infinite-dimensional vector space $E$, useful results have been obtained by imposing on $E$ the linear topology $t(V)$ described by Fischer and Gross [4], [5], [6], and investigated by Gross and Miller [9]. It has been shown that, in the induced topology, the Clifford algebra $C(E)$ is a topological algebra, but that, for topologies strictly finer than $t(V)$, multiplication need not be continuous. The main result of the present paper asserts that, even for topologies finer than $t(V)$, desirable conclusions can be drawn if continuity is replaced by hypocontinuity (see [2] for definition).

Introduction. In [4], [5], and [6], Fischer and Gross studied quadratic forms on infinite-dimensional vector spaces. Little had been known about this subject, since the algebraic methods customarily used to study forms on finitedimensional spaces fail when the dimension is infinite. It was found that, by imposition on the space $E$ of a topology canonically associated with the quadratic form, useful results could be obtained. The linear topologies of Lefschetz are especially suited to the task and, indeed, are nontrivial only for infinite dimensional E. Gross and Miller continued the work [9], discussing the Clifford algebra $C(E)$, and found that if $E$ is given the topology $t(V)$ described below, then, in the induced topology, $C(E)$ is a topological algebra. However, an example is given in [9] of a topology $t$ on $E$, with $t$ strictly finer than $t(V)$, for which even the tensor algebra $T(E)$ is not a topological algebra.

In $\$ 2$ it is shown that, even if $t$ is strictly finer than $t(V)$, multiplication is hypocontinuous, and some desirable conclusions may be drawn.

1. Preliminaries. We state here, for completeness, some known results that can be found in the above-mentioned works.

Received by the editors April 9, 1974.

AMS (MOS) subject classifications (1970). Primary 54A10, 54F65, 54B25.

Key words and phrases. Clifford algebra, linear topologies, hypocontinuity. 
Let $E$ be a vector space over a field $k$, with the characteristic of $k$ other than 2. Throughout this work, $k$ will have the discrete topology, and $E$ is topologized so that a base of neighborhoods at zero consists of linear subspaces. Such a topology $t$ on $E$ is called a linear topology, and has the property that every finite-dimensional, separated, linearly topologized space is discrete. If $\left(L_{i}\right)_{i \in I}$ and $F$ are linearly topologized spaces, then a multiline ar map $m: X_{i} L_{i} \rightarrow F$ is continuous if and only if it is separately continuous and continuous at zero. A subspace $L$ is linearly bounded if, for each zero-neighborhood $U, L \cap U$ is of finite codimension in $L$. Linear boundedness is preserved by continuous linear maps.

Let $q$ be a quadratic form on $E$ and $b$ its as sociated bilinear map on $E \times E$. If $A$ is a nonempty subset of $E$, then $A^{\perp}=\{x \in E: b(a, x)=0$ for all $a \in A\}$ is the orthogonal of $A$ with respect to $b$. The topology $t(b)$ is defined on $E$ by taking as a zero-neighborhood basis the orthogonals $U=D^{\perp}$ of finite-dimensional subspaces $D$ of $E$. This topology has the property that it is the coarsest linear topology on $E$ which makes the form $b$ separately continuous.

A subspace $F$ of $E$ is called totally isotropic with respect to $b$ if $q$ is identically zero on $F$. By Zorn's lemma each totally isotropic subspace $F$ is contained in a maximal such space $H$. The set $\{H \cap U: U$ is a $t(b)$ zero-neighborhood and $H$ is maximal totally isotropic $\}$ is a filterbase on $E$ and generates a linear topology which is denoted by $t(H)$. If $q$ is nondegenerate then the topologies $t(H)$ are separated, and the quadratic form $q$ is $t$ continuous if and only if there exists a maximal totally isotropic space $H$ such that $t$ is not coarser than $t(H)$.

On the tensor product of two vector spaces, a topology corresponding to the projective tensor product topology described by Grothendieck in [8] has been used: Let $(E, t)$ and $(F, s)$ be separated linear topological vector spaces over $k$. In [G] it is shown that there is a unique topology, denoted $t \otimes s$, on $E \otimes F$ such that

(i) the canonical bilinear map $b^{\prime}: E \times F \rightarrow E \otimes F$ is continuous;

(ii) if $G$ is a topological $k$-vector space, and the bilinear map $b$ : $E \times F \rightarrow G$ is continuous, then its corresponding linear map $1: E \otimes F \rightarrow G$ is continuous.

If $E^{n}$ denotes the tensor product of $E$ with itself $n$ times, then the tensor algebra $T(E)$ of $E$ is the direct sum $\bigoplus_{n=0}^{\infty} E^{n}$, where $E^{0}=k$ and $E^{1}=E$. For a quadratic form $q$ on $E$, let $I$ be the two-sided ideal generated by the elements of the form $x \otimes x-1 \cdot q(x)$ in $T(E)$. Then the 
Clifford algebra $C(E)$ is the quotient $T(E) / I$. If the summands of $T(E)$ are each given the projective topology $\bigotimes_{t}$, then the Clifford algebra can be topologized by extending through sums and quotients. These induced topologies will still be written $\bigotimes_{t}$.

The following propositions are due to Gross and Miller [9].

Proposition 1.1. Let $(E, t, q)$ be a linearly topologized space with quadratic form $q$. Then the following are equivalent:

(i) $\bigotimes_{t}$ restricted to $E$ is the original topology $t$;

(ii) $t \geq t(V)$ for some totally isotropic subspace $V$ of $E$;

(iii) the ideal $I$ is closed in $T(E)$;

(iv) $\left(C(E), \bigotimes_{t}\right)$ is Hausdorff.

Proposition 1.2. Multiplication is separately continuous in $\left(T(E), \bigotimes_{t}\right)$.

Proposition 1.3. If $E$ is of denumerable dimension and $V$ is an orthogonally closed totally isotropic subspace of $E$, then $\left(T(E), \bigotimes_{t}(V)\right)$ and $(C(E), \otimes t(V))$ are topological algebras, i.e. multiplication in $T(E)$ and $C(E)$ is continuous.

2. Hypocontinuity. Since we want $\otimes_{t}$ to be a generalization of $t$, Proposition 1.1 shows that only topologies not coarser than $\bigotimes_{t}$ are of interest. It is shown in [9] that a topology $t$, strictly finer than $t(V)$, can be imposed on a denumerable dimension $E$ such that $(T(E), \bigotimes t)$ is not a topological algebra. However, some desirable properties are obtained even for $t$ strictly finer than $t(V)$ if continuity is replaced by hypocontinuity. The reader is referred to [3], [4], and [6] for a detailed development of the notion of hypocontinuity of a multilinear map. We give the definition only in the case of a bilinear map: Let $E_{1}, E_{2}$ and $F$ be linear topological vector spaces over $k$. Let $G_{1}$ and $G_{2}$ be sets of closed subspaces of $E_{1}$ and $E_{2}$, respectively. A bilinear map $b: E_{1} \times E_{2} \rightarrow F$ is called $G_{1}$-hypocontinuous when the following are satisfied:

(i) $b$ is separately continuous;

(ii) for each zero-neighborhood $V$ in $F$ and each choice of a fixed $S$ in $G_{1}$, there is a zero-neighborhood $U$ in $E_{2}$ such that $b(S \times U)$ is a subset of $V$.

The definition for $G_{2}$-hypocontinuity is symmetric.

As an example, the evaluation map $\left(x, x^{*}\right)$ in $E \times E^{*}$, for $E^{*}$ the algebraic dual of $E$, is separately continuous but not continuous. However, it is 
hypocontinuous with respect to bounded subsets of $E$ and equicontinuous subsets of $E^{*}$.

We will show that for $t \geq t(V)$, multiplication is $G$-hypocontinuous on $T(E)$, if $G$ is the set of linearly bounded subsets of $T(E)$.

Recall that a topological inductive $\operatorname{limit}(E, t)=\underline{\lim }\left(E_{i}, t_{i}\right)$ is said to be strict if $E_{i} \subset E_{j}$ for $i<j$ and if the topology induced by $t_{j}$ on $E_{i}$ is $t_{i}$.

Proposition 2.1. Let $E=E_{1} \subset E_{2} \subset E_{3} \subset \ldots$ be a strict topological inductive limit, with each containment proper, and let $B$ be a linearly bounded subspace of $E$. Then $B$ is contained in $E_{m}$ for some $m$, and is linearly bounded there.

Proof. Let $B$ be linearly bounded in $E$ and suppose for a contradiction that $B$ is contained in no $E_{i}$. We will conclude that $B$ is not linearly bounded by constructing a zero-neighborhood $U$ such that $B /(B \cap U)$ is of infinite dimension.

Let $\left(i_{n}\right)_{n \geq 1}$ be an infinite sequence of distinct indices such that, for each $n, B \cap\left(E_{i_{n+1}} \backslash E_{i_{n}}\right)$ is nonempty. Such a sequence exists, for if $\left(i_{n}\right)_{n \geq 1}$ is not cofinal in $\left.{ }^{(i}\right)_{i \geq 1}$, then $B$ is contained in one of the $E_{i}$. For simplicity in notation, we write $(n)$ for $\left(i_{n}\right)$, and all subscripts in the proof refer to this sequence.

Let $V_{1}$ be a proper zero-neighborhood of $E_{1}$. Then, without loss of generality, there is some $x_{1}$ in $B \cap E_{1}$ with $x_{1}$ not in $V_{1}$; for if $B \subseteq V_{1}$ we may choose another $V_{1}$ (unless $E_{1}$ is a subset of $B$, in which case we may choose another $E_{1}$; for $B$ is not all of $E$ ).

Since the limit is strict, there is a $V_{2}$ in $E_{2}$ with $E_{1} \cap V_{2}=V_{1}$. Now $B \cap\left(E_{2} \backslash V_{2}\right)$ is nonempty for the following reasons: $x_{1}$ is in $B$ and $x_{1}$ is not in $E_{1} \cap V_{2}$; moreover, if $B$ is a subset of $V_{2}$, then $B$ is a subset of $V_{1}$, but we chose otherwise, so there is an element $x_{2}$ in $E_{2} \backslash E_{1}$ with $x_{2}$ in $B$ and $x_{2}$ not in $V_{2}$. Note that $x_{1}$ and $x_{2}$ are linearly independent since $x_{2}$ is in $E_{2} \backslash E_{1}$. Continuing, we obtain a sequence $\left(V_{n}\right)_{n=1}^{\infty}$, and setting $U=\bigcup_{n=1}^{\infty} V_{n}$, we have a zero-neighborhood in $E$. Also, we have a sequence $\left(x_{i}\right)_{i=1}^{\infty}$ in $B$ with no $x_{i}$ contained in $U$. The space spanned by this sequence of elements is of countably infinite dimension since the $\left(x_{i}\right)$ are linearly independent, and is contained in $B /(B \cap U)$. This shows that the assumption was false, and so $B$ is a subset of $E_{m}$ for some $m$.

Certainly $B$ is linearly bounded in $E_{m}$, for if not there is a zeroneighborhood $U_{m}$ in $E_{m}$ with $B /\left(B \cap U_{m}\right)$ of infinite dimension. But there is a neighborhood $U$ in $E$ with $U_{m}=U \cap E_{m}$ and hence $B \cap U=B \cap U_{m}$, 
so $B$ is not linearly bounded in $E$, a contradiction concluding the proof.

Letting $E^{i}$ denote the tensor product of $E$ with itself $i$ times and giving $E$ the topology $t \geq t(V)$ for some fixed totally isotropic subspace $V$ of $E$, then the tensor algebra $T(E)$ is the strict inductive limit of the partial sums $\bigoplus_{i=1}^{n} E^{i}, n=1,2, \ldots$. We can now state the main theorem.

Theorem 2.2. Let $G$ be the set of linearly bounded subsets of $T(E)$. Then multiplication $\otimes: T(E) \times T(E) \rightarrow T(E)$ is G-hypocontinuous.

Proof. Let $B$ be linearly bounded and let $U$ be a zero-neighborhood in $T(E)$. We will construct a zero-neighborhood $V$ in $T(E)$ such that $V \otimes B$ is in $U$. From Proposition $2.1, B$ is a subset of $\bigoplus_{i=1}^{n} E^{i}$ for some $n$, and since $B$ is bounded, then $B /(B \cap U)$ is finite-dimensional, say of dimension $m$. Let $b_{1}, b_{2}, \cdots, b_{m}$ be a basis for the $m$-dimensional supplement to $B \cap U$ in $B$. Then each $b$ in $B$ can be written in the form $b=\sum_{i=1}^{m} a_{i} b_{i}+$ $u$, where $u$ is in $B \cap U$ (and hence in $\bigoplus_{i=1}^{n} E^{i}$ ). Multiplication is separately continuous, so for each $i=1,2, \ldots, m$, there is a zero-neighborhood $W_{i}$ in $T(E)$ such that $W_{i} \otimes b_{i}$ is a subset of $U$.

A characterization of the zero-neighborhoods in the projective topology is given in [9]. The neighborhood $U$ has a representation

$$
\begin{aligned}
U=U_{1} & +U_{2} \otimes U_{2}+\sum\left[e_{2}\right] \otimes U_{2}\left(e_{2}\right)+U_{3} \otimes U_{3} \otimes U_{3} \\
& +\cdots+\sum\left[e_{p}^{1}\right] \otimes\left[e_{p}^{2}\right] \otimes \cdots \otimes\left[e_{p}^{p-n}\right] \otimes\left(U_{p}\left(e_{p}^{1} \cdots e_{p}^{p-n}\right)\right)^{n}+\cdots
\end{aligned}
$$

where the $e$ 's run through all elements in $E,[e]$ is the space spanned by $e$, and the U's run through a zero-neighborhood basis for $E$. The $\Sigma$ signifies $\Sigma_{e_{1} \cdots e_{r} \epsilon E} \Sigma_{\text {perms }}$, where for example,

$$
\sum_{e_{2} \in E} \sum_{\text {perms }}\left[e_{2}\right] \otimes U_{2}\left(e_{2}\right)=\sum_{e_{2} \in E}\left[e_{2}\right] \otimes U_{2}\left(e_{2}\right)+\sum_{e_{2} \in E} U_{2}\left(e_{2}\right) \otimes\left[e_{2}\right] .
$$

Now $B \cap U$ sits in a finite part of this sum, which we call

$$
U^{\prime}=U_{1}+\cdots+\sum\left[e_{p}^{1}\right] \otimes \cdots \otimes\left[e_{p}^{p-n}\right] \otimes\left(U_{p}\left(e_{p}^{1} \cdots e_{p}^{p-n}\right)\right)^{n} .
$$

We define

$$
V_{m}=\left(\bigcap_{i=1}^{n} U_{i}\right) \cap\left(\bigcap_{r=1}^{n}\left(\bigcap_{s=1}^{r} U_{r}\left(e_{1} \cdots e_{r-s}\right)\right)\right)
$$

and

$$
V_{m}\left(e_{1} \cdots e_{m-k}\right)=U_{m}\left(e_{1} \cdots e_{m-k}\right) \cap V_{m} .
$$

That is, all of the (finitely many) subscripted $U^{\prime}$ 's in $U^{\prime}$ are intersected with 
each of the subscripted $U^{\prime}$ 's in $U$. Now take

$$
V^{\prime}=V_{1}+V_{2} \otimes V_{2}+\sum\left[e_{2}\right] \otimes V_{2}\left(e_{2}\right)+V_{3} \otimes V_{3} \otimes V_{3}+\cdots
$$

and take $V=\left(\bigcap_{i=1}^{m} W_{i}\right) \cap V^{\prime}$.

To show that $V \otimes B$ is contained in $U$, let $v$ belong to $V$ and $b$ to $B$. Then $b=\sum_{i=1}^{m} a_{i} b_{i}+u$, so that $v \otimes b=\left(\sum_{i=1}^{m} a_{i}\left(v \otimes b_{i}\right)\right)+v \otimes u$. Since $v$ is in $W_{i}$ for all $i$, then $\sum_{i=1}^{m} a_{i}\left(v \otimes b_{i}\right)$ is an element of $U$, and we may focus our attention on $v \otimes u$. Recalling that $v$ is in $V$ and $u$ is in $U \cap\left(\bigoplus_{i=1}^{n} E^{i}\right)$ $=U^{\prime}$, and that each subscripted $V$ is a subset of each subscripted $U$, it is easy to see by investigating the forms of the factors in the product $V \otimes U^{\prime}$ that $v \otimes u$ lies in $U$. For example, $\left(V_{i} \otimes \ldots \otimes V_{i}\right) \otimes\left(U_{j} \otimes \ldots \otimes U_{j}\right)$ is contained in $\left(U_{j}\right)^{i+j}$, one of the factors in $U$, since $V_{i}$ is a subset of $\bigcap_{k=1}^{n} U_{k}$ which is a subset of $U_{j}$.

Continuing in this manner completes the proof.

We have not been able to obtain a strictly analogous result in $C(E)$. In order to pass to the conclusion that multiplication on $C(E)$ be $\bar{G}$-hypocontinuous for $\bar{G}$ the set of linearly bounded subspaces of $C(E)$, we need the result that each linearly bounded set in $C(E)$ can be realized as the canonical image of a linearly bounded set in $T(E)$. In a similar setting in the theory of locally convex spaces, it is known that the desired lemma is not true (cf. [14]). We settle for the following useful result.

Lemma 2.3. The composition $T(E) \times T(E) \stackrel{m}{\longrightarrow} T(E) \stackrel{p}{\longrightarrow} T(E) / I=C(E)$ is $G$-hypocontinuous and constant on equivalence classes modulo $I$.

Proof. Let $U$ be a zero-neighborhood in $C(E)$ and let $B$ be linearly bounded in $T(E)$. Then there is a zero-neighborhood $V$ in $T(E)$ such that $p(V)$ is contained in $U$, and a zero-neighborhood $W$ in $T(E)$ such that $m(W \times B)=W \otimes B$ is contained in $V$. Then $p(m(W \times B))=p(W \otimes B)$ is in $U$, establishing the $G$-hypocontinuity.

Next, we know that $p(a \otimes b)=p(a) \cdot p(b)$. Now if $a^{\prime}$ is in the same class as $a$, and $b^{\prime}$ is in the same class as $b$, then

$$
p(a \otimes b)=p(a) \cdot p(b)=p\left(a^{\prime}\right) \cdot p\left(b^{\prime}\right)=p\left(a^{\prime} \otimes b^{\prime}\right),
$$

giving the second part of the lemma.

We may impose a further restriction and obtain an imperfect analogue to Theorem 2.2 for $C(E)$. The easy proof is omitted.

Theorem 2.4. Let $G^{\prime}$ denote the set of subspaces $B^{\prime}$ in $C(E)$ for which $B^{\prime}=p(B)$ for some linearly bounded set $B$ in $T(E)$. Then multipli- 
cation $\bar{m}: C(E) \times C(E) \rightarrow C(E)$ is $G^{\prime}$-hypocontinuous.

A bilinear map $b: E_{1} \times E_{2} \rightarrow F$ is called $(G, H)$-hypocontinuous if $b$ is $G$-hypocontinuous in the first variable and $H$-hypocontinuous in the second variable, for $G$ and $H$ appropriate classes of sets. The theorems above are notationally symmetric, and so we can say that $m$ is $(G, G)$ hypocontinuous and that $\bar{m}$ is $\left(G^{\prime}, G^{\prime}\right)$-hypocontinuous. We now adapt several theorems on hypocontinuity, found in [4], to our present situation.

Proposition 2.5. If $S$ is a linearly bounded subspace of $T(E)$, then $m$ is continuous on $S \times T(E)$. If $S^{\prime}$ is in $G^{\prime}$ then $\bar{m}$ is continuous on $S^{\prime} \times$ $C(E)$.

Corollary 2.6. $m$ is uniformly continuous on each product $S \times T$, with $S$ and $T$ in $G . \bar{m}$ is uniformly continuous on each product $S^{\prime} \times T^{\prime}$, with $S^{\prime}$ and $T^{\prime}$ in $G^{\prime}$.

Proposition 2.7. If $S$ and $T$ are linearly bounded in $T(E)$, then $S \otimes T$ is linearly bounded in $T(E)$. If $S^{\prime}$ and $T^{\prime}$ are in $G^{\prime}$, then $S . T$ is bounded in $C(E)$.

We conclude with the remark that the methods used in the main results of $\$ 2$, as well as the prerequisites of $\$ 1$, relied heavily on the denumerability of the dimension of $E$. More information is now known concerning the Clifford algebra $C(E)$ for $E$ of nondenumerable dimension. See, for example, the paper [11] of H. A. Keller.

\section{REFERENCES}

1. N. Bourbaki, Eléments de mathématique. I: Les structures fondamentales de l'analyse. Fasc. VII, Livre II: Chap. 3: Algèbre multilinéaire, Actualités Sci. Indust., no. 1044, Hermann, Paris, 1948; 2nd ed., 1958. MR 10 \#231; 30 \# 104.

2. - Eléments de mathématique. XVIII. Part I. Les structures fondamentales de l'analyse. Livre V: Espaces vectoriels topologiques. Chaps. 3, 4, 5 , Actualités Sci. Indust., no. 1229, Hermann, Paris, 1955. MR 17, 1109.

3. - Eléments de mathematique. XXIV. Part I. Les structures fondamentales de l'analyse. Livre II: Chap. 9: Formes sesquilinéaires et formes quadratiques, Actualités Sci. Indust., no. 1272, Hermann, Paris, 1959. MR 21 \#6384.

4. H. Fischer and H. Gross, Quadratic forms and linear topologies. I, Math. Ann. 157 (1964), 296-325. MR 30 \#3149.

5. - - Quadratic forms and linear topologies. II: Non-real fields $k$ and infinite-dimensional k-vector spaces, Math. Ann. 159 (1965), 285-308. MR 32 \#142;

6. - Tensorprodukte linearer Topologien, Math. Ann. 160 (1965), 1-40. MR 32 \# 1538.

7. W. Greub, Multilinear algebra, Die Grundlehren der math. Wissenschaften, B and 136, Springer-Verlag, New York, 1967. MR 37 \#222.

8. A. Grothendieck, Produits tensoriels topologiques et espaces nucléaires, Mem. Amer. Math. Soc. No. 16 (1955). MR 17, 763. 
9. H. Gross and V. Miller, Quadratic forms and linear topologies. IV: Continuous forms in infinite-dimensional spaces, Comment. Math. Hel v. 42 (1967), 132-170. MR $35 \# 6709$.

10. O. Hamara, Quadratic forms on linearly topologized vector spaces, Portugal. Math. 27 (1968), 15-30. MR $41 \# 8448$.

11. H. Keller, Stetigke its fragen bei lineartopologischen Cliffordalgebren, Juris Verlag, Zurich, 1971.

12. G. Köthe, Topologische linear Räume. I, Die Grundlehren der math. Wissenschaften, Band 107, Springer-Verlag, Berlin 1960; English transl., Topological vector spaces, Die Grundlehren der math. Wissenschaften, Band 159, SpringerVerlag, New York, 1969. MR 40 \# 1750.

13. A. Robertson and W. Robertson, Topological vector spaces, Cambridge Tracts in Math. and Math. Phys., no. 53, Cambridge University Press, New York, 1964. MR 28 \#5318.

14. H. Schaefer, Topological vector spaces, Macmillan, New York, 1966. MR 33 \# 1689.

DEPARTMENT OF MATHEMATICS, COLLEGE OF THE VIRGIN ISLANDS, KINGSHILL, BOX 84, ST. CROIX, U.S.V.I. 00850 